Pacific Journal of Mathematics

FORMAL POWER SERIES

D DARRELl CASHWELL AND C. J. EVERt 


\section{FORMAL POWER SERIES}

\section{E. D. CAshwell and C. J. Everett}

Introduction. It has been shown [2] that the set $\Omega$ of all arithmetic functions $\alpha$ on $N=\{1,2,3, \cdots\}$ to the complex field $C$ is a unique factorization domain under ordinary addition and the "arithmetic" product:

$$
(\alpha \beta)(n)=\Sigma \alpha(d) \beta(n / d) .
$$

The proof was based on the obvious isomorphism between $\Omega$ and the domain $C\left[\left[x_{1}, x_{2}, \cdots\right]\right]$ of formal power series over $C$, in countably many variables, induced by the mapping

$$
\alpha \rightarrow \Sigma \alpha\left(p_{1}^{a_{1}} p_{2}^{a_{2}} \cdots\right) x_{1}^{a_{1}} x_{2}^{a_{2}} \cdots,
$$

and the fact that the domain $C\left[\left[x_{1}, \cdots, x_{n}\right]\right]$ of such series in any finite number of variables is factorial (i.e., a unique factorization domain).

Recently D. Buchsbaum [1] and P. Samuel [8] have shown that the latter domains are factorial whenever $C$ is a regular factorial domain, in particular, a principal ideal ring.

It therefore seems appropriate to generalize our previous result in the following way. We replace the integers $n$, which in standard form are uniquely defined by their sequences $a_{1}, a_{2}, \cdots$ of exponents, by vectors $a$, finitely nonzero on an arbitrary set $I$ of indices $i$, and consider the corresponding ring $\Omega$ of functions defined on the set of all such vectors to an arbitrary domain of integrity $C$.

The analogue of the above mapping establishes an isomorphism between $\Omega$ and the ring $P$ of formal power series over $C$ in the variables $x_{i}, i \in I$. We prove that these rings, $\Omega$ and $P$, are factorial if and only if all domains $C\left[\left[x_{1}, \cdots, x_{n}\right]\right]$ are factorial.

It follows from the theorem of Buchsbaum and Samuel that $P$ is factorial whenever the coefficient domain is a regular factorial domain. Specializing to the case of a countable set of indices, we see that the ring of integer-valued arithmetic functions is factorial (cf. [9]; p. 36). Indeed this is true if the complex field $C$ is narrowed to the integral domain $R[\Theta]$ of any algebraic number field provided $R[\Theta]$ is itself factorial [7; p. 99].

These results appear as corollaries of a general theorem on Gaussian semi-groups to which we devote the second part of the paper.

1. The ring $\Omega$ of functions. Let $I$ be an arbitrary infinite set of

Received May 9, 1962. 
"indices" $i$. We refer only to finite subsets of $I$, among which we include the null set $\Theta$.

The set $E$ consists of the nonnegative integers $0,1,2, \cdots$, which play the rôle of exponents.

An arbitrary function $a$ on $I$ to $E$ which is nonzero on any finite subset $D(a)$ of $I$ is called a vector. The set of all such vectors is denoted by $N$. For the zero vector, $D(0)=\Theta$. The sum of two vectors, defined in the usual way, is again a vector. We shall say $a$ vector $a$ is inside $F$ in case $D(a) \subset F$, otherwise, $a$ is outside $F$.

Let $C$ be an arbitrary domain, i.e., a commutative, associative ring with identity element 1 , satisfying the condition: $x \neq 0 \neq y$ implies $x y \neq 0$.

Finally, $\Omega$ is taken to be the set of all functions $\alpha$ on $N$ to $C$. In $\Omega$ we define sum $\alpha+\beta$ and product $\alpha \beta$ by the identities (for all $c \in N)$ :

$$
\begin{gathered}
(\alpha+\beta)(c)=\alpha(c)+\beta(c) \in C, \\
(\alpha \beta)(c)=\Sigma \alpha(a) \beta(b) \in C,
\end{gathered}
$$

where the summation $\Sigma$ extends over all ordered pairs, $a, b$ of vectors such that $a+b=c$. Since $c(i)=a(i)+b(i)$, it is clear that $a$ and $b$ are inside $D(c)$ and have values $\leqq c(i)$ at each $i \in D(c)$. Hence only a finite number of vector pairs $a, b$ have sum $c$, and the above summation is well-defined in $C$.

Thus $\Omega$ is closed under the stated operations, and indeed we have

THEOREM 1. $\Omega$ is a domain.

A proof that $\Omega$ is a ring may be modelled in an obvious way on that indicated in [2]. The identity element $\varepsilon$ of $\Omega$ is the function defined by $\varepsilon(0)=1, \varepsilon(a)=0$ for all vectors $a \neq 0$. That $\Omega$ has no proper divisors of zero will appear in $\S 4$ with less trouble than a direct proof at this point.

2. The ring $P$ of power series. Let $P=C\left[\left[x_{i} ; i \in I\right]\right]$ be the set of all formal power series

$$
A=\Sigma \alpha(a) I I x_{i}{ }^{a(i)},
$$

where the sum extends over all vectors a of $N, \alpha$ is any fixed function on $N$ to $C$, and for each $a$, the product extends over all $i \in I$. If

$$
B=\Sigma \beta(b) I I x_{i}{ }^{b(i)}
$$

is another such series, and $A+B$ and $A B$ are defined formally, the latter subject to the "collection of like terms", we obtain again series 
in $P$. In particular

$$
A B=\Sigma(\Sigma \alpha(a) \beta(b)) I I x_{i}{ }^{c(i)},
$$

the first sum extending over all vectors $c$, the second over all pairs $a, b$ with $a+b=c$.

It is manifest that the correspondence

$$
\alpha \rightarrow A
$$

defines an isomorphism

$$
\Omega \cong P
$$

so that the latter is also a ring. To be sure, we might deal throughout exclusively with $P$; it is only for the sake of simplicity that we suppress the dummy $x_{i}$ and treat, for the most part, the ring $\Omega$ which we have introduced.

3. The $F$-subrings of $\Omega$. Let $F$ be an arbitrary finite subset of $I, \Theta$ included, and consider the totality $\Omega^{F}$ of all those elements $\alpha$ of $\Omega$ which are zero-valued for every vector a outside $F$. For example, $\Omega^{\theta}$ consists of those functions $\alpha$ which are zero-valued for every $a \neq 0$, and

$$
\Omega^{\circledast} \cong C
$$

under the correspondence $\alpha \rightarrow \alpha(0)$ on $\Omega^{\theta}$ to $C$.

If we return to the isomorphism

$$
\Omega \cong P
$$

it becomes clear that the defining correspondence $\alpha \rightarrow A$ induces an isomorphism between $\Omega^{F}$ and a set of series which is itself isomorphic to the domain $C\left[\left[x_{1}, \cdots, x_{n}\right]\right]$ of formal power series over $C$ in $n$ variables, $n$ being the cardinal of $F$. In case $F=\Theta$, we obtain of course $C$ itself. Thus we have

TheOREM 2. Under the correspondence

$$
\alpha \rightarrow A
$$

we have

$$
\Omega \cong P
$$

Moreover,

$$
\Omega^{F} \cong C\left[\left[x_{1}, \cdots, x_{n}\right]\right]
$$


for every finite subset $F$ of $n$ elements.

Thus the sets $\Omega^{F}$ are themselves domains under the operations of $\Omega$.

4. The $F$-projections. Once more, let $F$ be a finite set of indices, and for each $\alpha$ of $\Omega$, define the " $F$-projection" of $\alpha$ to be that element $F(\alpha)$ of $\Omega$ such that $F(\alpha)(a)=\alpha(a)$ for all vectors a inside $F$, and $F(\alpha)(\alpha)=0$ for all vectors outside $F$.

We devote the present section to deriving some formal properties $P 0, \cdots, P 7$ of these operators. Obviously we have

P0. $F(\alpha) \in \Omega^{F}$.

It is easily seen that $F(\alpha+\beta)=F(\alpha)+F(\beta)$ by comparing the values of these two functions at vectors $c$ inside and outside $F$.

Similarly we establish

P1. $\quad F(\alpha \beta)=F(\alpha) F(\beta)$.

For, $(F(\alpha) F(\beta))(c)=\Sigma F(\alpha)(a) \cdot F(\beta)(b)$ where $a+b=c$. For $c$ inside $F$, the summands $a$ and $b$ are also, and the latter sum becomes $\Sigma \alpha(a) \beta(b)=(\alpha \beta)(c)$, which agrees with $F(\alpha \beta)(c)$. If $c$ is outside $F$ and $a+b=c$, either $a$ or $b$ is outside $F$ and we have both sides of $P 1$ zero-valued.

Thus we see that $\alpha \rightarrow F(\alpha)$ defines a ring homomorphism: $\Omega \sim \Omega^{F}$.

We obtain trivially at this point that $\Omega$ is a domain of integrity. If $\alpha \neq 0 \neq \beta$ in $\Omega$, there exist vectors $a, b$ such that $\alpha(a) \neq 0 \neq \beta(b)$ in $C$. Let $F=D(a) \cup D(b)$. Since $a$ and $b$ are inside $F$,

$$
F(\alpha)(a)=\alpha(a) \neq 0 \neq \beta(b)=F(\beta)(b) .
$$

Thus $F(\alpha)$ and $F(\beta)$ are nonzero elements of $\Omega^{F} \cong C\left[\left[x_{1}, \cdots, x_{n}\right]\right]$, a well-known domain. Consequently (using $P 1$ ), $F(\alpha \beta)=F(\alpha) F(\beta) \neq 0$. Then there is a vector $c$ such that $F(\alpha \beta)(c) \neq 0$. Necessarily $c$ is inside $F$, and so $(\alpha \beta)(c) \neq 0$. Hence $\alpha \beta \neq 0$.

It is clear from the definitions of $\Omega^{F}$ and $F(\alpha)$ that

P2. $\alpha \in \Omega^{F}$ implies $F(\alpha)=\alpha$.

Moreover, we have the monotone property:

P3. For $F \subset G, F(G(\alpha))=F(\alpha)$.

For a vector outside $F$, both sides have value zero, while for $c$ inside $F$, we have $F(\alpha)(c)=\alpha(c)$ and also $F(G(\alpha))(c)=G(\alpha)(c)=\alpha(c)$ since $D(a) \subset F \subset G$.

By a unit of a domain is meant a divisor of the identity. We 
prove the less trivial

P4. If $\Theta(\alpha)$ is a unit of $\Omega^{\theta}$, then $\alpha$ is a unit of $\Omega$.

From the isomorphism $\alpha \rightarrow \alpha(0)$ of $\Omega^{\theta}$ to $C$ it is clear that $\Theta(\alpha)(0)=\alpha(0)$ is a unit of $C$. We have to show the existence of an element $\beta$ in $\Omega$ such that $\alpha \beta=\varepsilon$, that is to say,

$$
\begin{aligned}
& 1=\varepsilon(0)=(\alpha \beta)(0)=\alpha(0) \beta(0) \\
& 0=\varepsilon(c)=(\alpha \beta)(c)=\Sigma \alpha(a) \beta(b)
\end{aligned}
$$

where $a+b=c \neq 0$.

We define $\beta(c)$ inductively on the length $\lambda(c)=\Sigma c(i)$. The only vector of length zero is 0 , and we define $\beta(0)=1 / \alpha(0)$ in $C$. Suppose $\beta(c)$ already defined for all vectors $c$ of length $\lambda(c)<l$, where $l$ is any fixed integer $>0$. For each vector $c$ of length $l$, define

$$
\beta(c)=-\beta(0) \Sigma \alpha(\alpha) \beta(b)
$$

summed over all $a, b$ such that $a+b=c$ and $b \neq c$. All such vectors $b$ are of length $\lambda(b)<\lambda(c)=l$, so $\beta(c)$ is well-defined, and the function $\beta$ so constructed satisfies $\alpha \beta=\varepsilon$.

We next note the uniqueness property

P5. If $\alpha, \beta \in \Omega$, and $K(\alpha)=K(\beta)$ for all $K \supset J$, then $\alpha=\beta$.

Let $a$ be an arbitrary vector and set $K=J \cup D(a)$. Obviously $\alpha(a)=K(\alpha)(a)=K(\beta)(a)=\beta(a)$.

By a "sequence over $J$ " we mean a function which assigns to every finite set $K \supset J$ a definite element $\delta^{K}$ of $\Omega^{K}$. Such a sequence is called projective in case $K\left(\delta^{L}\right)=\delta^{K}$ whenever $J \subset K \subset L$. Our domain $\Omega$ is complete in the sense of

P6. Every projective sequence $\delta^{K}$ over $J$ is of the form

$$
\delta^{K}=K(\delta)
$$

for some $\delta$ of $\Omega$.

Define $\delta(a)=\delta^{K}(a)$, where $K=J \cup D(a)$. Fix $L \supset J$. Clearly $L(\delta)(a)=\delta^{L}(\alpha)$ for $a$ outside $L$, while for $D(a) \subset L$ we have

$$
K=J \cup D(a) \subset J \cup L=L,
$$

so that

$$
L(\delta)(a)=\delta(a)=\delta^{K}(a)=K\left(\delta^{L}\right)(a)=\delta^{L}(a) .
$$

We shall require also the following analogue of $P 6$ which asserts the completeness of the system of associate classes. 
P7. For every sequence $\delta^{F}$ over $\Theta$ such that $\delta^{F} \sim F\left(\delta^{\sigma}\right)$ in $\Omega^{F}$ for all $F \subset G$, there exists a $\delta \in \Omega$ such that $F(\delta) \sim \delta^{F}$ in $\Omega^{F}$ for every $F$.

Here $\alpha \sim \beta$ signifies $\alpha=\beta \nu$ for some unit $\nu$ of the indicated domain. This well-known equivalence relation splits a domain into disjoint classes $[\alpha]$ of "associates". For example [0] consists of the single element 0 , while the associates of the identity are precisely the units of the domain.

If every $\delta^{F}=0$ in the given sequence the result is trivial with $\delta=0$. For $F(0)=0$ for every $F$ by $P 2$.

On the other hand, suppose some $\delta^{J} \neq 0$. We have then for every $K \supset J$ also that $\delta^{K} \neq 0$. For, $J\left(\delta^{K}\right) \sim \delta^{J} \neq 0$ in $\Omega^{J}$ and $J(0)=0$. If we exhibit a $\delta \in \Omega$ such that $K(\delta) \sim \delta^{K}$ for all $K \supset J$, this will serve. For if $F$ is arbitrary and we set $K=J \cup F$, we shall have

$$
F(\delta)=F(K(\delta))=F\left(\nu^{K} \delta^{K}\right)=F\left(\nu^{K}\right) F\left(\delta^{K}\right),
$$

where by assumption $F\left(\delta^{K}\right) \sim \delta^{F}$ in $\Omega^{F}$. Now $\nu^{K}$ is a unit of $\Omega^{K}$, and from $\nu^{K} \mu^{K}=\varepsilon$ follows $F\left(\nu^{K}\right) F\left(\mu^{K}\right)=F(\varepsilon)=\varepsilon$ so that $F\left(\nu^{K}\right)$ is a unit of $\Omega^{F}$. Thus we shall have $F(\delta) \sim \delta^{F}$ in $\Omega^{F}$ for every $F$.

We have therefore to prove the following: Given a sequence $\delta^{K}$ over $J$ such that, for every $J \subset K \subset L, 0 \neq \delta^{K}=\nu_{L}^{K} K\left(\delta^{L}\right)$, where $\nu_{L}^{K}$ is a unit of $\Omega^{K}$ dependent on the pair $K \subset L$, there exists a $\delta \in \Omega$ such that $K(\delta)=\nu^{K} \delta^{K}$ for all $K \supset J$, where $\nu^{K}$ is a unit of $\Omega^{K}$.

We first note that the given units satisfy the transitivity condition

$$
\nu_{M}^{K}=\nu_{L}^{K} K\left(\nu_{M}^{L}\right) \text { for } \quad J \subset K \subset L \subset M .
$$

To see this, we substitute $\delta^{L}=\nu_{M}^{L} L\left(\delta^{M}\right)$ into the preceding equation for $\delta^{K}$ to obtain $\delta^{K}=\nu_{L}^{K} K\left(\nu_{H^{L}}^{L}\right) K\left(\delta^{M}\right)$, using $P 1, P 3$. Comparing this with the direct relation $\delta^{K}=\nu_{M}^{K} K\left(\delta^{M}\right)$ and remembering that $\delta^{K}$, and hence $K\left(\delta^{M}\right)$, is nonzero, we obtain $(T)$ by cancellation.

In view of $P 6$, it suffices to define a sequence $\nu^{K}$ of units such that the transformed sequence $\nu^{K} \delta^{K}$ is projective over $J$. For this a sequence of units $\nu^{K}$ over $J$ satisfying

$$
\nu^{K} \nu_{L}^{K}=K\left(\nu^{L}\right) \text { for } \quad J \subset K \subset L
$$

will serve. For this would yield $K\left(\nu^{L} \delta^{L}\right)=K\left(\nu^{L}\right) K\left(\delta^{L}\right)=\nu^{K} \nu_{L}^{K} K\left(\delta^{L}\right)$ $=\nu^{K} \delta^{K}$.

Our problem may therefore be regarded as that of obtaining from a given "double sequence" of units $\nu_{L}^{K}$ satisfying $(T)$ a "single sequence" $\nu^{K}$ satisfying $\left(T^{\prime}\right)$. Note that $\nu_{K}^{K}=\varepsilon$ from $(T)$ so that $\left(T^{\prime}\right)$ is trivial for $K=L$.

We proceed to define the desired sequence $\nu^{K}, K \supset J$ inductively on the number of elements $k$ by which the (finite) set $K$ exceeds $J$. 
For $K=J(k=0)$, define $v^{J}=\varepsilon$. For a set $K$ of one more index than $J(k=1)$, set $\nu^{K}=\nu_{K}^{J}$. This is an element of $\Omega^{J} \subset \Omega^{K}$, and is a unit of $\Omega^{J}$, hence also of $\Omega^{K}$. Note that all $\nu^{K}$ so far defined satisfy $\left(T^{\prime}\right)$, since $J\left(\nu^{K}\right)=J\left(\nu_{K}^{J}\right)=\nu_{K}^{J}=\varepsilon \nu_{K}^{J}=\nu^{J} \nu_{K}^{I}$.

Now let $m$ be an arbitrary integer $>1$, and assume $\nu^{K}$ already defined and satisfying $\left(T^{\prime}\right)$ for all $K \supset J$ with $k<m$. Let $M$ be any fixed set over $J$ with $m$ more indices than $J$.

We define $\nu^{M}$ at every vector $a$ as follows: For $a$ outside $M$, $\nu^{M}(a)=0$. For a vector $a$ with $D(a) \subset M$, set $K \equiv J \cup D(a) \subset J \cup M=M$. If $K=M$, set $\nu^{M}(a)=0$. Finally, if $K$ is a proper subset of $M, \nu^{K}$ is already defined, and we set $\nu^{M}(a)=\left(\nu^{K} \nu_{M}^{K}\right)(a)$.

The function $\nu^{M}$ thus defined is certainly in $\Omega^{M}$, and since

$$
D(0)=\Theta \subset M, \text { and } K \equiv J \cup \Theta=J
$$

is a proper subset of $M(m>1)$, we have defined $\nu^{M}(0)$ as $\left(\nu^{J} \nu_{M}^{J}\right)(0)$. This is clearly a unit of $C$, so $(P 4) \nu^{M}$ is a unit of $\Omega$, hence also of $\Omega^{M}$. (Note: from $\nu^{M} \mu=\varepsilon$ in $\Omega$ follows $\nu^{M} M(\mu)=M(\varepsilon)=\varepsilon$ in $\Omega^{M}$, by $P 1, P 2, P 0$.)

Verification of $\left(T^{\prime}\right)$ at the $m$-level requires only that $L\left(\nu^{M}\right)(a) \equiv$ $\left(\nu^{L} \nu_{M}^{L}\right)(a)$ for each $L$ over $J$ which is a proper subset of $M, \nu^{M}$ being the unit just defined. This is trivial for a outside $L$. Hence let $D(a) \subset L$. We have then to show $\mathcal{\nu}^{M}(a)=\left(\mathcal{L}^{L} \nu_{M}^{L}\right)(a)$. Now

$$
K=J \cup D(a) \subset J \cup L=L,
$$

properly in $M$. We defined $\nu^{M}(\alpha)=\left(\nu^{K} \nu_{M}^{K}\right)(a)$. On the other hand, $\nu^{K}$ and $\nu^{L}$ are already known to satisfy $\left(T^{\prime}\right): \nu^{K} \nu_{L}^{K}=K\left(\nu^{L}\right)$, and we are given from $(T)$ that $\nu_{M}^{K}=\nu_{L}^{K} K\left(\nu_{M}^{L}\right)$. Thus we see that $K\left(\nu^{L} \nu_{M}^{L}\right)=$ $K\left(\nu^{L}\right) K\left(\nu_{M}^{L}\right)=\nu^{K} \nu_{L}^{K} K\left(\nu_{M}^{L}\right)=\nu^{K} \nu_{M}^{K}$. But

$$
D(a) \subset K \text {, so }\left(\nu^{K} \nu_{M}^{K}\right)(a)=K\left(\nu^{L} \nu_{M}^{L}\right)(a)=\left(\nu^{L} \nu_{M}^{L}\right)(a) .
$$

Thus $\left(T^{\prime}\right)$ still holds at the $m$-level and all is proved.

5. Abstract foundation of the factorial problem. For simplicity we have suppressed the "variables" $x_{i}$ of $P$ and have derived certain basic properties of the isomorphic domain $\Omega$, which show that its elements under multiplication constitute a system of the kind we now discuss.

In introducing this abstraction, obviously suggested by the power series case, we are motivated not simply by a striving for greater generality, but by a natural desire for clarity and economy of means.

The type of system natural for the general problem of unique factorization is the commutative semi-group [5; Ch. IV] with identity and cancellation law. Since the $F$-projection of nonzero elements of 
a power series ring may well be zero, it is preferable from our point of view to study what we may call, for want of a name, binary domains. These are trivially related to the more familiar semi-groups, and a system of either kind is obtainable from the other in an obvious way.

Let us say then that a set $\Omega$ of elements $\alpha$ is a binary domain if it possesses a commutative, associative, binary operation $\alpha \beta$, an identity $\varepsilon(\varepsilon \alpha \equiv \alpha)$ and a zero $0 \neq \varepsilon(0 \alpha \equiv 0)$, and if, finally, it satisfies the cancellation law: $\alpha \beta=\alpha \gamma$ implies $\beta=\gamma$ whenever $\alpha \neq 0$.

In such a system, $\alpha \neq 0 \neq \beta$ obviously implies $\alpha \beta \neq 0$. Moreover, a subset of such a system, which is itself a binary domain under the given operation, necessarily has for its zero and identity those of the system itself.

The divisors of the identity $\varepsilon$ in a binary domain $\Omega$ are called units and constitute an abelian group $\Upsilon$ with identity $\varepsilon$. As usual the relation $\alpha \sim \beta$, meaning $\alpha=\beta \nu$ for some $\nu \in \Upsilon$, defines disjoint classes $[\alpha]$ of associates in $\Omega$.

Let $\mathscr{F}=\{F\}$ be a directed set with null-element $\Theta$, i.e., a partially ordered set with an element $\Theta \leqq F$ for all $F \in \mathscr{F}$, and having for every pair $F, G$ in $\mathscr{F}$ an element $H \in \mathscr{F}$ such that $H \geqq F$ and $H \geqq G$.

Assume further that there exists an $\mathscr{F}$-indexed class of subsets $\Omega^{F}$ of $\Omega, F \in \mathscr{F}$, which are themselves binary domains under the given operation. We denote the corresponding groups of units by $\gamma^{F}$.

Finally we suppose every $F \in \mathscr{F}$ defines on $\Omega$ a specific mapping $F(\alpha)$, so written for simplicity, such that the following axioms hold:

A0. $F(\alpha) \in \Omega^{F}$;

A1. $\quad F(\alpha \beta)=F(\alpha) F(\beta)$;

A2. $\alpha \in \Omega^{F}$ implies $F(\alpha)=\alpha$;

A3. $F \leqq G$ implies $F(G(\alpha))=F(\alpha)$;

A4. $\Theta(\alpha) \in \Upsilon^{\theta}$ implies $\alpha \in \Upsilon$;

A5. $F(\alpha)=F(\beta)$ for all $F$ implies $\alpha=\beta$;

A6. Every sequence $\delta^{F} \in \Omega^{F}$ with $F\left(\delta^{\theta}\right)=\delta^{F}$ for all $G \geqq F \geqq \Theta$ is of form $\delta^{F}=F(\delta)$;

A7. For every sequence $\delta^{F} \in \Omega^{F}$ with $F\left(\delta^{\theta}\right) \sim \delta^{F}$ in $\Omega^{F}$ whenever $G \geqq F \geqq \Theta$, there exists a $\delta \in \Omega$ such that $F(\delta) \sim \delta^{F}$ in $\Omega^{F}$ for every $F$.

We shall prove the following

Principal Theorem. A binary domain $\Omega$ satisfying all of the preceding conditions is factorial if and only if every $\Omega^{F}$ is factorial.

Since the multiplicative systems of our power series domains have 
been shown to satisfy these conditions (the set $\mathscr{F}$ consisting of the finite subsets $F$ of the index set $I$ ) and since their subdomains $\Omega^{F}$ are (even ring-) isomorphic to the power series domains $C\left[\left[x_{1}, \cdots, x_{n}\right]\right]$ (cf. Theorem 2) we may cite the immediate consequences:

CoRollary 1. The domain $P$ of formal power series over a domain of integrity $C$, in an arbitrary set of variables, is factorial if and only if the series domains $C\left[\left[x_{1}, \cdots, x_{n}\right]\right]$ are factorial for every finite $n$.

Corollary 2. Every domain $P$ of formal power series over a regular factorial domain $C$ is factorial.

For $C\left[\left[x_{1}, \cdots, x_{n}\right]\right]$ is factorial whenever $C$ is a regular factorial domain [1], [8].

COROLlaRY 3. The domain of "arithmetic functions" with values in any subfield of the complex field $C$, or in any factorial algebraic number domain $R[\Theta]$ of degree $n \geqq 1$ is factorial.

For these value domains are all principal ideal rings. [7; p. 99] We turn to a proof of the principal theorem.

6. Immediate consequences of the axioms. We first derive some simple lemmas.

L1. $F(0)=0$ and $F(\varepsilon)=\varepsilon$ for every $F$. For 0 and $\varepsilon$ are in every $\Omega^{F}$, as we have remarked, and so A2 suffices.

L2. $\Omega^{F} \cap \Upsilon=\Upsilon^{F}$.

If $\alpha \beta=\varepsilon$, where $\alpha \in \Omega^{F}, \beta \in \Omega$, then $\alpha F(\beta)=F(\alpha) F(\beta)=F(\alpha \beta)=$ $F(\varepsilon)=\varepsilon$ by A2, A1, L1, and from A0, $F(\beta) \in \Omega^{F}$. The opposite inclusion is immediate from the definition of units.

L3. If $\gamma \neq 0$ in $\Omega$, there exists a $J$ such that $K(\gamma) \neq 0$ for all $K \geqq J$.

If $F(\gamma)=0$ for every $F$, we have $\gamma=0$ from L1 and A5. If $J(\gamma) \neq 0$ and $K \geqq J$, then by $\mathrm{A} 3, J(K(\gamma))=J(\gamma) \neq 0$, so $K(\gamma) \neq 0$ by L1.

L4. If $\beta \in \Upsilon$, then $F(\beta) \in \Upsilon^{F}$ for every $F$. If $\beta \in \Omega$ and for some $F, F(\beta) \in \Upsilon^{F}$, then $\beta \in \Upsilon$.

From $\alpha \beta=\varepsilon$ in $\Omega$ follows $F(\alpha) F(\beta)=\varepsilon$ by A1, L1, and $F(\beta) \in$ $\Upsilon^{F}$ by $\mathrm{A} 0$.

From $\alpha F(\beta)=\varepsilon$ with $\alpha \in \Omega^{F}$ follows $\Theta(\alpha) \Theta(\beta)=\varepsilon$ by A1, A3, L1. Since $\Theta(\beta) \in \Upsilon^{\theta}, \beta \in \Upsilon$ (A4).

L5. If $K(\alpha)=K(\beta)$ for all $K \geqq J$ then $\alpha=\beta$.

For $F$ arbitrary, take $K \geqq J, F$, Then (A3) $F(\alpha)=F(K(\alpha))=$ 
$F(K(\beta))=F(\beta)$, and we cite A5.

Similarly we generalize A6. The reader may note that adopting L5, L6 as axioms would have established direct connection with P5, $\mathrm{P} 6$ of series domains. Although it involves a little extra work we have preferred the simpler axioms.

L6. Every projective sequence $\gamma^{K}$ over $J$ is of the form $\gamma^{K}=K(\delta)$.

Let $F$ be arbitrary, and take $K \geqq J, F$. Tentatively, define $\delta^{F}=F\left(\gamma^{K}\right)$. If also $L \geqq J, F$, we see that $F\left(\gamma^{K}\right)=F\left(\gamma^{L}\right)$. For take $M \geqq K, L$. Since $\gamma^{K}$ is projective over $J$, we have $\gamma^{K}=K\left(\gamma^{M}\right)$ and $\gamma^{L}=L\left(\gamma^{M}\right)$. From A3, we have $F\left(\gamma^{K}\right)=F\left(\gamma^{M}\right)=F\left(\gamma^{L}\right)$, since $M \geqq K \geqq$ $F$, and $M \geqq L \geqq F$. Thus $\delta^{F}$ is well-defined.

We show the full sequence $\delta^{F}$ is projective. For $G \geqq F$, we have to prove $F\left(\delta^{G}\right)=\delta^{F}$. Take $K \geqq J, G$. Since $G \geqq F$, also $K \geqq J, F$. Hence we may write both $\delta^{F}=F\left(\gamma^{K}\right)$ and $\delta^{G}=G\left(\gamma^{K}\right)$. From A3 follows $F\left(\delta^{G}\right)=F\left(\gamma^{K}\right)=\delta^{F}$. From A6, we have $\delta^{F}=F(\delta)$ for all $F$, $\delta$ fixed in $\Omega$.

In particular, for $K \geqq J, \delta^{K}=K(\delta)$. Since $K \geqq J, K$, we have by definition (and A2) $\delta^{K}=K\left(\gamma^{K}\right)=\gamma^{K}$. Hence $\gamma^{K}=K(\delta), K \geqq J$, and $\delta$ serves.

Finally we prove in analogous fashion the following consequence of $\mathrm{A} 7$.

L7. If $\gamma^{K} \in \Omega^{K}$ is a sequence over $J$ such that $K\left(\gamma^{L}\right) \sim \gamma^{K}$ in $\Omega^{K}$ whenever $L \geqq K \geqq J$, then there exists $a \delta \in \Omega$ such that $K(\delta) \sim \gamma^{K}$ in $\Omega^{K}$ for all $K \geqq J$.

First suppose $J, F \leqq U, V \leqq M$. Then $U\left(\gamma^{M}\right)=\nu^{U} \gamma^{U}, \quad V\left(\gamma^{M}\right)=$ $\nu^{V} \gamma^{V}$, so by A3, A1, $F\left(\nu^{V}\right) F\left(\gamma^{\sigma}\right)=F\left(\gamma^{M}\right)=F\left(\nu^{V}\right) F\left(\gamma^{V}\right)$. Here $\nu^{U} \in \gamma^{U}$ and $\nu^{v} \in \Upsilon^{\nu}$. It follows from $\mathrm{L} 2$ and $\mathrm{L} 4$ that $F\left(\nu^{\sigma}\right)$ and $F\left(\nu^{v}\right)$ are units of $\Omega^{F}$ and hence $F\left(\gamma^{\sigma}\right) \sim F\left(\gamma^{\nabla}\right)$ in $\Omega^{F}$.

Now for each $F$ let $U=t(F)$ be any particular $U \geqq J, F$, where we take $t(K)=K$ in case $K \geqq J$, and define $\delta^{F}=F\left(\gamma^{\sigma}\right)$.

For $G \geqq F$, set $V=t(G) \geqq J, G$. Since $V \geqq J, F$ also, we have from the first paragraph that $F\left(\gamma^{\sigma}\right) \sim F\left(\gamma^{V}\right)$ in $\Omega^{F}$. Hence $F\left(\delta^{A}\right)=$ $F\left(G\left(\gamma^{V}\right)\right)=F\left(\gamma^{\nabla}\right) \sim F\left(\gamma^{U}\right)=\delta^{F}$ in $\Omega^{F}(\mathrm{~A} 3)$. By A7, there is a $\delta \in \Omega$ such that $F(\delta) \sim \delta^{F}$ in $\Omega^{F}$ for every $F$.

In particular $K(\delta) \sim \delta^{K}$ in $\Omega^{K}, K \geqq J$. But we defined $t(K)=K$, so that $\delta^{K}$ is by definition $K\left(\gamma^{K}\right)=\gamma^{K}(\mathrm{~A} 2)$, and $K(\delta) \sim \gamma^{K}, K \geqq J$.

7. The chain condition. An arbitrary binary domain is said to satisfy the chain condition in case

Axıom C. Every sequence $\cdots \gamma_{2}\left\|\gamma_{1}\right\| \gamma_{0}$ of proper divisors is finite. 
Here $\alpha \| \gamma$ means $0 \neq \gamma=\alpha \beta$, where the (uniquely defined) $\beta$ is not a unit.

It is well-known that Ax. C implies

(F) Every nonzero non-unit element is expressible as a product of a finite number $(\geqq 1)$ of irreducible elements.

(An irreducible element $\pi$ is a nonzero non-unit element such that $\pi=\alpha \beta$ implies $\alpha$ or $\beta$ a unit.)

Returning to our domain $\Omega$, we have

THeOREm 3. The chain condition holds in $\Omega$ if and only if it holds in all $\Omega^{F}$.

If $0 \neq \gamma=\alpha \beta$, where $\gamma, \alpha \in \Omega^{F}$ and $\beta$ is a non-unit of $\Omega^{F}$, then $\beta \notin \Upsilon$ by L2.

If $0 \neq \gamma=\alpha \beta$, where $\gamma, \alpha \in \Omega$, and $\beta$ is a non-unit of $\Omega$, then by A1, $0 \neq J(\gamma)=J(\alpha) J(\beta)$ where $J$ is the index of L3, and $J(\beta) \notin Y^{J}$ by L4.

Thus a proper divisor chain in $\Omega^{F}$ is such a chain in $\Omega$, while a chain in $\Omega$ induces one in the indicated $\Omega^{J}$.

8. Uniqueness of factorization. An arbitrary binary domain is called factorial in case it satisfies condition $(\mathrm{F})$ of the previous section and also

(U) If $\pi_{1} \cdots \pi_{m}=\tau_{1} \cdots \tau_{n}$, where all factors are irreducible, then $m=n$ and the $\pi_{i}$ are associates of the $\tau_{j}$ under some permutation.

Of the various conditions insuring (U) the simplest for our purpose is

Aхіом P. If $0 \neq \pi \gamma=\alpha \beta$, where $\pi$ is irreducible then $\pi \mid \alpha$ or $\pi \mid \beta$.

The well-known result on which we rely is therefore the equivalence of Axioms $\mathrm{C}$ and $\mathrm{P}$ in a domain with the propositions ( $\mathrm{F}$ ) and (U).

Returning again to our system $\Omega$, we note first the existence of irreducibles of a very simple type.

L8. If $\alpha \in \Omega$, and for some $J, J(\alpha)$ is an irreducible element of $\Omega^{J}$, then $\alpha$ is irreducible in $\Omega$, and indeed $K(\alpha)$ is an irreducible element of $\Omega^{K}$ for all $K \geqq J$.

By definition, $J(\alpha) \neq 0, J(\alpha) \notin \Upsilon^{J}$, so that $\alpha \neq 0$ and $\alpha \notin \Upsilon$ by L1, L4. Suppose $\alpha=\beta \gamma$ in $\Omega$. By A1, $J(\alpha)=J(\beta) J(\gamma)$ with factors in $\Omega^{J}$ (A0); $J(\alpha)$ being irreducible in $\Omega^{J}$, we have (say) $J(\beta) \in \Upsilon^{J}$. Then $\beta \in \Upsilon$ by L4, and $\alpha$ is irreducible in $\Omega$.

For $K \geqq J$, we have (A3) $J(K(\alpha))=J(\alpha) \neq 0$, whence $K(\alpha) \neq 0$, (L1). Since $\alpha \notin \gamma, K(\alpha) \notin \gamma^{K}$ (L4). Suppose $K(\alpha)=\beta \gamma ; \beta, \gamma \in \Omega^{K}$. Then (A3, A1), $J(\alpha)=J(\beta) J(\gamma)$ with factors in $\Omega^{J}(\mathrm{~A} 0)$. Again we 
have (say) $J(\beta) \in \Upsilon^{J}$, whence $\beta \in \Upsilon^{\curvearrowright} \cap \Omega^{K}=\Upsilon^{K}$ (L4, L2).

From this it is easy to infer

THEOREM 4. If Axiom $P$ holds in $\Omega$ it holds in every $\Omega^{F}$. Thus all $\Omega^{F}$ are factorial in case $\Omega$ is factorial.

Let $0 \neq \pi \gamma=\alpha \beta ; \gamma, \alpha, \beta \in \Omega^{F}, \pi$ an irreducible element of $\Omega^{F}$. By A2, $\pi=F(\pi)$, and $\pi$ is an irreducible element of $\Omega$ by the preceding lemma. From Axiom $\mathrm{P}$ in $\Omega$ we conclude (say) $\alpha=\pi \delta$ for some $\delta \in \Omega$. Thus $\alpha=F(\alpha)=F(\pi) F(\delta)=\pi F(\delta)$ by A1, A2, and $F(\delta) \in \Omega^{F}$ by A0.

Thus Axiom $\mathrm{P}$ holds in $\Omega^{F}$, and the theorem follows from the appropriate part of Theorem 3 .

9. The fundamental lemma. We have now established the easy implication of the principal theorem, and turn to the converse. To be sure, in view of Theorem 3, it would suffice to show that Axiom $\mathrm{P}$ in all $\Omega^{F}$ implies this axiom in $\Omega$. Unfortunately we are unable to follow so simple a course. Instead we shall prove, on the assumption that all $\Omega^{F}$ are factorial (satisfying Axiom $\mathrm{C}$ and Axiom $\mathrm{P}$ ) that the irreducible elements of $\Omega$ are necessarily of the simple type encountered in L8. We state this fundamental lemma (proved in \$11) and show at once how it leads quickly to our final goal.

L9. If all $\Omega^{F}$ are factorial, and $\gamma$ is an irreducible element of $\Omega$, then there exists an $F$ for which $F(\gamma)$ is an irreducible element of $\Omega^{F}$.

Suppose this to be true, and let us deduce the final requirement for the principal theorem:

THEOREM 5. If every $\Omega^{F}$ is factorial, then $\Omega$ is factorial.

We need only verify Axiom $\mathrm{P}$ in $\Omega$. Hence let $0 \neq \gamma \pi=\alpha \beta ; \alpha, \beta$, $\gamma \in \Omega, \pi$ an irreducible element of $\Omega$. From L8 and L9 there exists a $J$ such that $M(\pi)$ is irreducible for all $M \geqq J$. Since $\gamma \neq 0$, we have also (L3) a $K$ such that $M(\gamma) \neq 0$ for $M \geqq K$. By the directed set property, there is an $L \geqq J, K$, and so we have for all $M \geqq L$, $0 \neq M(\pi) M(\gamma)=M(\alpha) M(\beta)$ in $\Omega^{M}$, with $M(\pi)$ irreducible in $\Omega^{M}$. Since the latter is factorial, $M(\pi)$ divides either $M(\alpha)$ or $M(\beta)$ in $\Omega^{M}, M \geqq L$.

Let $C(\alpha)$ be the set of all $M \geqq L$ for which the first eventuality obtains, with $C(\beta)$ similarly defined. These sets may well overlap but their union certainly contains every $M \geqq L$. We claim at least one of these two sets is co-final with $\mathscr{F}$, that is to say, for every $M \geqq L$ there exists in this set a $P \geqq M$. For, if each set fails in this respect, there would be an $R \geqq L$, not $\leqq$ any element of $C(\alpha)$, 
and an $S \geqq L$, not $\leqq$ any element of $C(\beta)$. But there is a $T \geqq R$, $S \geqq L$, so $T$ must belong to $C(\alpha)$ or $C(\beta)$. If $T \in C(\alpha)$, then $R \leqq T$ contradicts the choice of $R$, with a similar contradiction if $T \in C(\beta)$.

Let us suppose then that $C(\alpha)$ is co-final with $\mathscr{F}$. For every $M \geqq L$, we have a $P \geqq M$ such that $P(\alpha)=P(\pi) \delta^{P}$ where $\delta^{P} \in \Omega^{P}$. This implies $M(\alpha)=M(\pi) M\left(\delta^{P}\right)$ in $\Omega^{M}$, by A1, A3, A0. We may therefore write for every $M \geqq L, M(\alpha)=M(\pi) \delta^{M}$, where $\delta^{M} \in \Omega^{M}$.

We shall verify that $\delta^{M}$ is a projective sequence over $L$. For, if $P \geqq M$, it follows (by A1, A3) from $P(\alpha)=P(\pi) \delta^{P}$ that $M(\alpha)=$ $M(\pi) M\left(\delta^{P}\right)$. Since $M(\pi) \neq 0$ (it is irreducible in $\Omega^{M}$ ), we obtain $\delta^{M}=$ $M\left(\delta^{P}\right)$ for all $P \geqq M \geqq L$. It follows from L6 that $\delta^{M}=M(\delta), M \geqq L$, for some $\delta \in \Omega$. So we have $M(\alpha)=M(\pi) M(\delta)=M(\pi \delta), M \geqq L$, whence $\alpha=\pi \delta$ by L5, which completes the proof.

10. A set theoretic principle. We shall appeal in the following section to a theorem on sets for which we have no definite reference. Perhaps its direct proof can best be based on an obvious rewording of that given in [3] for a special case concerned with the representation of sets. An indication of such a proof may be found in the final section. It may also be regarded as a consequence (with discrete topology) of a theorem of Tychonoff $[6 ; \mathrm{p} .19]$ to the effect that an arbitrary product of compact spaces is compact. (In this connection see also [4]). In any case, one seems to need Zorn's lemma or its equivalent at this point.

Our only purpose here is to state the theorem in a form adapted to immediate use.

Let $\left[M_{K}, T\right]$ be an indexed class of finite non-null sets $M_{K}, K \in T$. Let $R$ be a collection of finite choice functions $s=\{s(K) ; S\}$, where $s(K) \in M_{K}$ for all $K \in S \subset T, S$ being a finite set of indices $K$, and suppose that $R$ is complete in the sense that

A. If $s$ is in $R$, and $S_{1}$ is any subset of its domain $S$, then the sub-function defined by $s$ on $S_{1}$ is also in $R$.

B. Every finite set $S$ of indices is the domain of at least one $s$ of $R$.

Zorn's lemma then implies the existence of a choice function $t=\{t(K) ; T\}$ defined for all indices $K \in T$ such that, for every finite set $S \subset T$, the sub-function defined by $t$ on $S$ is in $R$.

11. Proof of the fundamental lemma. The elements of a binary domain are of four mutually exclusive kinds: zero, units, irreducible elements, and composite elements. Thus a composite element $\gamma$ always has a true factor, that is to say, a non-unit proper divisor. Since 
all associates of a true factor of $\gamma$ are also true factors we may speak of an associate class of true factors of $\gamma$. In the sequel we shall be dealing with associate classes in the systems $\Omega^{K}$ and shall always mean by $\left[\delta^{K}\right]$ the set $\Upsilon^{K} \delta^{K}$ of associates of $\delta^{K} \in \Omega^{K}$ in $\Omega^{K}$.

Turning to L9, suppose all $\Omega^{F}$ are factorial, and $\gamma$ is an irreducible element of $\Omega$. Since $\gamma \neq 0$ there exists (L3) a $J$, hereafter fixed, such that $K(\gamma) \neq 0$ for all $K \geqq J$. Moreover, $F(\gamma) \notin \Upsilon^{F}$ for any $F$ since $\gamma \notin \Upsilon$ (L4). Assume the conclusion of L9 false. Then for every $F$, we have $F(\gamma)=0$ or $F(\gamma) \in \gamma^{F}$ or $F(\gamma)$ composite in $\Omega^{F}$. Thus for every $K \geqq J, K(\gamma)$ is composite in $\Omega^{K}$ and this ring contains a class $\left[\delta^{K}\right]$ of true factors of $K(\gamma)$. The set $M_{K}$ of all such classes (considered as elements) is non-null and finite (since $\Omega^{K}$ is factorial). The totality of these $M_{K}$ constitutes a collection of sets, indexed on the set $T$ of all $K \geqq J$, of the kind introduced in $\S 10$.

Define $R$ to be the set of all finite choice functions $s=\{s(K) ; S\}$, $S$ finite $\subset T, s(K) \in M_{K}$, which have the property

(P) for every pair of indices $K, L$ in $S$ for which $K \leqq L$, there exist elements $\delta^{K} \in s(K), \delta^{L} \in s(L)$ such that $\delta^{K}=K\left(\delta^{L}\right)$.

We show that $R$ is complete in the sense of $\S 10$. Property (A) is sufficiently obvious. For (B), let $S=\{K\}$ be any finite set of indices in $T$ and take $L \geqq K$ for all $K \in S$. From the known factorization $L(\gamma)=\alpha^{L} \beta^{L}$ into true factors $\alpha^{L}, \beta^{L}$ in $\Omega^{L}$, we deduce (A3, A1, A0) for each $K \in S$ the factorization $K(\gamma)=K\left(\alpha^{L}\right) K\left(\beta^{L}\right)$ in $\Omega^{K}$, where neither factor is a unit of $\Omega^{K}$ by L4, L2. Hence we define

$$
s(K)=\left[K\left(\alpha^{L}\right)\right] \in M_{K}, K \in S .
$$

This is certainly a valid finite choice function on $S$. We verify (P). If $K$ and $K^{\prime}$ are in $S$, and $K \leqq K^{\prime}$, we have $K\left(K^{\prime}\left(\alpha^{L}\right)\right)=K\left(\alpha^{L}\right)$ by A3. Thus the set $R$ is complete.

We conclude from $\S 10$ the existence of a function $t(K)=\left[\gamma^{K}\right] \in M_{K}$ which assigns to every $K \geqq J$ a class $\left[\gamma^{K}\right]$ of true factors of $K(\gamma)$ in $\Omega^{K}$ such that, in particular, for every pair of indices $K \leqq L, \gamma^{K} \sim K\left(\gamma^{L}\right)$ in $\Omega^{K}$. Here the $\gamma^{K}$ is an arbitrarily chosen representative of the class $t(K)$, and property (P) gives us elements $\delta^{K}=\tau^{K} \gamma^{K}, \delta^{L}=\tau^{L} \gamma^{L}$ with $K\left(\delta^{L}\right)=K\left(\tau^{L}\right) K\left(\gamma^{L}\right)=\delta^{K}=\tau^{K} \gamma^{K}$ where $\tau^{K} \in \gamma^{K}, \tau^{L} \in \gamma^{L}$. Since $\tau^{L} \in Y^{L}=\Upsilon \cap \Omega^{L} \subset \Upsilon$ (L2), it follows from L4 that $K\left(\tau^{L}\right) \in Y^{K}$ and thus $\gamma^{K} \sim K\left(\gamma^{L}\right)$ as stated.

We write $K(\gamma)=\gamma^{K} \rho^{K}$ in $\Omega^{K}$ for every $K \geqq J$. From L7, there exists a $\delta \in \Omega$ such that $K(\delta)=\nu^{K} \gamma^{K}, \nu^{K} \in \Upsilon^{K}, K \geqq J$. If $\mu^{K}$ is the inverse of $\nu^{K}$ in $\gamma^{K}$, we have $K(\gamma)=K(\delta) \sigma^{K}$ in $\Omega^{K}$, where $\sigma^{K}=\mu^{K} \rho^{K}$.

The sequence $\sigma^{K}$ is projective over $J$. To see this, write $L(\gamma)=L(\delta) \sigma^{L}$ for $L \geqq K$. This gives $K(\gamma)=K(\delta) K\left(\sigma^{L}\right)$ by A3, A1. Since $K(\gamma) \neq 0$ $(K \geqq J)$, also $K(\delta) \neq 0$ and we obtain $K\left(\sigma^{L}\right)=\sigma^{K}$. By L6, there exists a $\sigma \in \Omega$ such that $\sigma^{K}=K(\sigma), K \geqq J$. Thus $K(\gamma)=K(\delta) K(\sigma)=K(\delta \sigma)$, 
$K \geqq J . \quad$ By L5, $\gamma=\delta \sigma$ in $\Omega$.

Now $K(\delta)=\nu^{K} \gamma^{K}$ and $K(\sigma)=\sigma^{K}=\mu^{K} \rho^{K}$ are non-units of $\Omega^{K}$. Hence $\delta$ and $\sigma$ are non-units of $\Omega$ (L4). Thus $\gamma$ is composite in $\Omega$. This contradiction establishes L9.

12. Completion for elements. For the purposes of this and the following section, let us call a binary domain satisfying all conditions of $\S 5$ except possibly $\mathrm{A} 6$ and $\mathrm{A} 7$ an $\mathscr{F}$-domain, and consider in place of A7 the following weaker condition.

A7*. For every sequence $\delta^{F} \in \Omega^{F}$ such that $\delta^{F} \sim F\left(\delta^{G}\right)$ in $\Omega^{F}$ whenever $\Theta \leqq F \leqq G$, there exists a sequence of units $\nu^{F} \in Y^{F}$ such that $\nu^{F} \delta^{F}$ is a projective sequence.

In the presence of $\mathrm{A} 6$ (and A3) this is trivially equivalent to A7.

Suppose now that $\Omega$ is an $\mathscr{F}$-domain and let us take $\Omega^{*}$ to be the set of all projective sequences $\left\{\alpha^{F}\right\}, \alpha^{F} \in \Omega^{F}, F\left(\alpha^{G}\right)=\alpha^{F}$ for all $\Theta \leqq F \leqq G$. It is easily verified that $\Omega^{*}$ is closed, and indeed a binary domain under the operation $\left\{\alpha^{F}\right\}\left\{\beta^{F}\right\}=\left\{\alpha^{F} \beta^{F}\right\}$. The zero and identity are of course the "constant" sequences $\{0\}$ and $\{\varepsilon\}$ respectively, and the units of $\Omega^{*}$ are the projective sequences $\left\{\nu^{F}\right\}, \nu^{F} \in \gamma^{F}$.

For example, the cancellation law may be established in the following manner. If $\left\{\alpha^{F}\right\} \neq\{0\}, \alpha^{J} \neq 0$ for some $J$, so for $K \geqq J, J\left(\alpha^{K}\right)=$ $\alpha^{J} \neq 0$ implies also $\alpha^{K} \neq 0$ since $J(0)=0$ (L1). Hence from $\left\{\alpha^{F}\right\}\left\{\beta^{F}\right\}=$ $\left\{\alpha^{F}\right\}\left\{\gamma^{F}\right\}$ we have $\beta^{K}=\gamma^{K}, K \geqq J$. For $F$ arbitrary, take $K \geqq J, F$ and note that $\beta^{F}=F\left(\beta^{K}\right)=F\left(\gamma^{K}\right)=\gamma^{F}$.

The subset $\Omega^{* K}$ of $\Omega^{*}$ consisting of all $F$-sequences of the form $\left\{F\left(\alpha^{K}\right)\right\}, \alpha^{K}$ arbitrary but fixed in $\Omega^{K}$, is itself a binary domain and the operations $K\left\{\alpha^{F}\right\}=\left\{F\left(\alpha^{K}\right)\right\}, K \geqq \Theta$ satisfy A0-A5.

For example, we note that the units of $\Omega^{* K}$ are the $F$-sequences $\left\{F\left(\nu^{K}\right)\right\}, \nu^{K} \in \gamma^{K}$. From this remark (for $K=\Theta$ ), A4 follows at once.

In addition, $\mathrm{A} 6$ holds in $\Omega^{*}$. Given a $K$-sequence of elements $\left\{F\left(\alpha^{K}\right)\right\}, \alpha^{K} \in \Omega^{K}$, such that $K\left\{F\left(\alpha^{L}\right)\right\}=\left\{F\left(\alpha^{K}\right)\right\}, K \leqq L$, we have for each such pair $K, L$ that $F\left(K\left(\alpha^{L}\right)\right)=F\left(\alpha^{K}\right)$ for all $F$. Thus the $F$-sequence $\left\{\alpha^{F}\right\}$ is projective by A5 and is therefore an element of $\Omega^{*}$ such that $K\left\{\alpha^{F}\right\}=\left\{F\left(\alpha^{K}\right)\right\}$ for all $K$.

Finally we observe that the mapping (*) $\alpha \rightarrow\{F(\alpha)\}$ is one-to-one (A5) on all $\Omega$ to a subset (A3) of $\Omega^{*}$, preserves multiplication (A1), and is a $K$-operator isomorphism in the sense that $K(\alpha) \rightarrow K\{F(\alpha)\}$. Moreover, it is of interest for the general theory that each subdomain $\Omega^{K}$ is isomorphic under $(*)$ to all of the corresponding $\Omega^{* K}$.

We summarize these remarks in

THeorem 6. An $\mathscr{F}$-domain $\Omega$ with subdomains $\Omega^{F}$ is always embeddable, with preservation of $\mathscr{F}$-projection, in a complete (A6) 
$\mathscr{F}$-domain whose subdomains $\Omega^{* F}$ coincide with those of $\Omega^{F}$. Moreover $A 7^{*}$ holds in $\Omega^{*}$ if and only if it holds in $\Omega$.

The final statement is trivial from the isomorphism $(*)$ and the "finite character" of A7*. From the principal theorem of $\$ 5$ follows the

CoRollary. An $\mathscr{F}$-domain $\Omega$ satisfying $A 7^{*}$ is embeddable in an $\mathscr{F}^{\mathrm{F}}$-domain $\Omega^{*}$ which is factorial if and only if all $\Omega^{F}$ of $\Omega$ are factorial. If such an $\Omega$ is factorial so is $\Omega^{*}$.

For we have shown in $\$ \S 7,8$ that all $\Omega^{F}$ of a factorial $\mathscr{F}$-domain $\Omega$ are also factorial; no use of A6 or A7 was made in this part of the proof of the principal theorem.

Finally, suppose $\Omega$ is an $\mathscr{F}$-domain satisfying $\mathrm{A} 7 *$ and has all $\Omega^{F}$ factorial. Then $\Omega^{*}$ provides a factorial extension of $\Omega$, and it is of some interest to note the following relations between $\Omega$ and $\Omega^{*}$ (the first two are true for any $\mathscr{F}$-domain).

(1) If any $\alpha^{J}$ of a projective sequence $\left\{\alpha^{F}\right\}$ is a unit (in $\left.\Omega^{J}\right)$ then $\left\{\alpha^{F}\right\}$ is a unit of $\Omega^{*}$.

For $F$ arbitrary, take $K \geqq J . F$. Since $J\left(\alpha^{K}\right)=\alpha^{J} \in Y^{J}, \alpha^{K} \in \Upsilon$ and $\alpha^{F}=F\left(\alpha^{K}\right) \in Y^{F}(\mathrm{~L} 4)$.

(2) If any $\alpha^{J}$ of a projective sequence $\left\{\alpha^{F}\right\}$ is an irreducible element of $\Omega^{J}$, then $\left\{\alpha^{F}\right\}$ is an irreducible element of $\Omega^{*}$.

For then $\left\{\alpha^{F}\right\}$ is a nonzero non-unit element of $\Omega^{*}$ and $\left\{\alpha^{F}\right\}=$ $\left\{\beta^{F}\right\}\left\{\gamma^{F}\right\}$ implies $\alpha^{J}=\beta^{J} \gamma^{J}$, whence $\beta^{J}$ or $\gamma^{J}$ is a unit of $\Omega^{J}$. So (2) follows from (1). (It is also clear that $\alpha^{K}$ is irreducible in $\Omega^{K}$ for all $K \geqq J$.)

(3) If $\left\{\alpha^{F}\right\}$ is irreducible in $\Omega^{*}$, there exists a $J$ such that $\alpha^{J}$ is irreducible in $\Omega^{J}$.

For L9 applied to $\Omega^{*}$ says that there exists a $J$ such that $J\left\{\alpha^{F}\right\}=$ $\left\{F\left(\alpha^{J}\right)\right\}$ is irreducible in $\Omega^{* J}$. Hence $\alpha^{J}$ is irreducible in $\Omega^{J}$ under the isomorphism $(*)$.

(4) Every nonzero non-unit element of $\Omega$ is expressible as a product of irreducible elements.

For Axiom $\mathrm{C}$ holds in all $\Omega^{F}$ and so also in $\Omega$, as we showed in $\S 7$. 
(5) An irreducible element $\pi$ of $\Omega$ remains irreducible in $\Omega^{*}$ if and only if it is finitely irreducible.

By this we mean that $\{F(\pi)\}$ is irreducible in $\Omega^{*}$ if and only if $J(\pi)$ is irreducible in $\Omega^{J}$ for some $J$ (cf. L9, L8), which is clear from (2) and (3).

(We do not know an example of an irreducible element $\pi$ with $F(\pi)$ composite in $\Omega^{F}$ for every $F$.)

(6) If $0 \neq \pi \gamma=\alpha \beta$ in $\Omega$, where $\pi$ is an irreducible element of the type in (5), then either $F(\pi) \mid F(\alpha)$ for every $F$ or $F(\pi) \mid F(\beta)$ for every $F$.

For $\Omega^{*}$ is factorial.

EXAMPLE. Let $\Omega^{\prime}$ be the domain isomorphic with the power series ring $P^{\prime}=C\left[\left[x_{1}, x_{2}, \cdots\right]\right]$ over the rational integers $C$ in a countable set of variables. Take $\Omega$ to be the subset of $\Omega^{\prime}$ obtained from it by deleting all units not in any $\Omega^{\prime F}$. This is tantamount to deleting from $P^{\prime}$ all units involving an infinite number of $x_{i}$. It is easy to verify that $\Omega$ is an $\mathscr{F}$-domain under multiplication which satisfies $\mathrm{A} 7^{*}$ but not A6. Moreover we still have the finite subdomains $\Omega^{F} \cong C\left[\left[x_{1}, \cdots, x_{n}\right]\right]$.

13. Completion for classes. Let $\Omega$ be an $\mathscr{F}$-domain satisfying A6. In the present section all square brackets refer to associate classes in $\Omega$ itself, that is, $[\alpha]$ means the set $\gamma \alpha$, regardless of the nature of $\alpha$ in $\Omega$. Moreover, we define $K[\alpha]=[K(\alpha)]=\gamma \cdot K(\alpha)$ and not $\gamma^{K} K(\alpha)$.

First consider the set $\Omega^{\dagger}$ of all associate classes of $\Omega$. This is obviously a binary domain under the well-defined operation $[\alpha][\beta]=$ $[\alpha \beta]$, and corresponds to the more familiar concept of reduced semigroup. The zero and identity are [0] and $[\varepsilon]=\gamma$ respectively, and the latter is the sole unit element of $\Omega^{\dagger}$.

The subset $\Omega^{\dagger}$ of all $\Omega$-classes $\left[\alpha^{K}\right], \alpha^{K} \in \Omega^{K}$, is a subdomain of $\Omega^{\dagger}$ whose only unit is $[\varepsilon]$ (L2).

It should be noted at this point that for two elements $\alpha^{K}$ and $\beta^{K}$ of $\Omega^{K}, \alpha^{K} \sim \beta^{K}$ in $\Omega$ if and only if $\alpha^{K} \sim \beta^{K}$ in $\Omega^{K}$, and that the welldefined correspondence $\left[\alpha^{K}\right] \rightarrow \Upsilon^{K} \alpha^{K}$ is an isomorphism between $\Omega^{\dagger K}$ and the internal classes of $\Omega^{K}$.

One easily verifies that $K$-projection as defined above on $\Omega^{\dagger}$ satisfies $\mathrm{A} 0-\mathrm{A} 5$. Consider in particular A5. Suppose $K[\alpha]=K[\beta]$, i.e., $K(\alpha) \sim K(\beta)$ in $\Omega$, for every $K$. If $\beta=0$, then $K(\beta)=0=K(\alpha)$ for all $K$, and $\alpha=0$ also (L1, A5). Hence $[\alpha]=[\beta]$. If $\beta \neq 0$, we have (L3) $K(\beta) \neq 0$ for all $K \geqq J$, and $K(\alpha)=\nu^{K} K(\beta)$, where the unit $\nu^{K}$ 
of $\Omega$ is necessarily in $\gamma^{k}$ (A2, $\left.\mathrm{A} 1, \mathrm{~A} 0, \mathrm{~L} 2\right)$. If we write also, for $L \geqq K, L(\alpha)=\nu^{L} L(\beta)$, and take $K$-projection of both sides, we see that the sequence $\nu^{K}, K \geqq J$, is projective. By L6, we may write $\nu^{K}=K(\nu)$ for some $\nu \in \Omega$, which is clearly a unit (L4). Thus $K(\alpha)=$ $K(\nu) K(\beta)=K(\nu \beta)$, and so $\alpha=\nu \beta$ (L5), whence $[\alpha]=[\beta]$.

THEOREM 7. The associate classes of an $\mathscr{F}$-domain $\Omega$ satisfying A6 form an $\mathscr{F}$-domain $\Omega^{\dagger}$. Moreover, $\Omega^{\dagger}$ satisfies A6 if and only if $\Omega$ satisfies $\mathrm{A} 7 *$.

If $\Omega$ satisfies $\mathrm{A}^{*}$ (and so all the axioms) then $\mathrm{A} 7$ holds in $\Omega$ (cf. Sec. 12). Let $\left[\delta^{K}\right]$ be a projective sequence of classes in $\Omega^{\dagger}$, i.e., for every $K \leqq L, K\left[\delta^{L}\right]=\left[\delta^{K}\right]$, so that $K\left(\delta^{L}\right) \sim \delta^{K}$ in $\Omega$. As we just saw, this implies $K\left(\delta^{L}\right) \sim \delta^{K}$ in $\Omega^{K}$, so from A7 we have a $\delta \in \Omega$ such that $K(\delta) \sim \delta^{K}$ in $\Omega^{K}$, hence also in $\Omega$, for every $K$. Thus $K[\delta]=\left[\delta^{K}\right]$ in $\Omega^{\dagger}$.

Conversely, assume A6 in $\Omega^{\dagger}$, and consider a sequence in $\Omega$ such that $K\left(\delta^{L}\right) \sim \delta^{K}$ in $\Omega^{K}$ (hence in $\Omega$ ) for all $K \leqq L$. From A6 in $\Omega^{\dagger}$ follows the existence of a class $[\delta]$ such that $K[\delta]=\left[\delta^{K}\right]$. Thus $K(\delta) \sim$ $\delta^{K}$ in $\Omega$, and so in $\Omega^{K}$, for all $K$. We therefore have A7 and A7* in $\Omega$.

It may be noted here that our axioms A0 - A7 were chosen simply to insure that the reduced system $\Omega^{\dagger}$ should indeed have the essential properties $\mathrm{A} 0-\mathrm{A} 6$, which formed the real basis for the principal theorem.

Finally, consider the case of an $\mathscr{F}$-domain satisfying A6, but not $\mathrm{A} 7^{*}$. The $\mathscr{F}$-domain $\Omega^{\dagger}$ then lacks $\mathrm{A} 6$ (A7* is trivially true in $\Omega^{\dagger}$ ). We may therefore embed $\Omega^{\dagger}$ in the domain $\Omega^{\dagger *}$ of sequences $\left\{\left[\alpha^{F}\right]\right\}$ according to the procedure of $\$ 12$. Thus we have the final

THEOREM 8. The domain $\Omega^{\dagger}$ of associate classes of an $\mathscr{F}$-domain $\Omega$ satisfying A6 can always be embedded, with preservation of projection, in an $\mathscr{F}$-domain $\Omega^{\dagger *}$ satisfying all the axioms, and whose $K$-subdomains coincide with those of $\Omega^{\dagger}$. Thus $\Omega^{* *}$ is factorial if and only if all $\Omega^{F}$ of $\Omega$ are factorial.

This follows at once from the theorem of $\S 12$ and the isomorphism between $\Omega^{\dagger K}$ and the internal classes of $\Omega^{K}$.

14. Proof of a theorem in set theory. For the sake of completeness we indicate a proof of the theorem in $\$ 10$, following exactly the argument given in [3] for a special case. We are given a complete set $R$ of finite choice functions $s=\{s(K), S\}$. Call a function $u=$ $\{u(K) ; U\}, u(K) \in M_{K}, K \in U$ (arbitrary) $\subset T$, universal (relative to $R$ ) in case, for every finite set $S \subset T$ there exists in $R$ a function $\{s(K) ; S\}$ 
which agrees with $u$ on $S \cap U$.

It is an easy matter to verify that the set of all universal functions is a non-null partially ordered set $P$, every linearly ordered subset $L$ of which has an upper bound in $P$.

Here the order relation $\{u(K) ; U\} \leqq\left\{u_{1}(K) ; U_{1}\right\}$ is taken to mean $U \subset U_{1}$ and $u(K) \equiv u_{1}(K)$ for $K \in U$. The upper bound referred to is obtained by taking for its domain the set union of all domains involved in $L$, and defining its value at any index of this domain (unambiguously) to be the value of any function of $L$ whose domain includes this index. It is trivial to verify that the function so constructed is itself universal.

That $P$ is non-void may be obtained by the stratagem of the "choice function defined on the empty set of indices", together with the completeness property (B) of $R$, or by following the procedure indicated in the final paragraph to construct universal functions on domains of one index.

Zorn's lemma states that every partially ordered set $P$ of the kind described in italics contains a maximal element.

Now one can show, using the finiteness of the sets $M_{K}$, that a universal function $u$ whose domain $U$ does not exhaust $T$ can be extended (see last paragraph) to a universal function whose domain is enlarged by an additional index. Thus the maximal element insured by Zorn's lemma must have total domain $T$, and being universal, each of its finite sub-functions is certainly in $R$.

To extend a universal function $u=\{u(K) ; U\}$ to a domain $U_{1}=$ $U \cup\{Q\}$, where $Q \notin U$, we argue thusly: If no universal function $u_{1}=\left\{u_{1}(K) ; U_{1}\right\} \geqq u=\{u(K) ; U\}$ exists, we should have, for every choice $u_{1}(Q)=q \in M_{Q}$, the existence of a finite set $S(q)$ (fix one) such that every function $s$ in $R$ with domain $S(q)$ disagrees with the proposed $\left\{u_{1}(K) ; U_{1}\right\}$ on $S(q) \cap U_{1}$. Since $u$ is universal, $S(q)$ certainly contains $Q$. Since $M_{Q}$ is a finite set and each $S(q)$ is a finite set of indices, so is the set union $S=\cup S(q), q \in M_{Q}$. But, $u$ being universal, there is some function $s$ in $R$ with domain $S$ which agrees with $u$ (on $U \cap S$ ). Since $Q \in S, s$ assigns to $Q$ some element $q^{*}=s(Q)$ of $M_{Q}$. The sub-function $s^{*}$ of $s$ defined by $s$ on $S\left(q^{*}\right)$ is itself in $R$ by property (A), it assigns $q^{*}$ to $Q$, and it agrees with $u$ on $S\left(q^{*}\right) \cap U$. This contradicts the nature of $S\left(q^{*}\right)$, and hence shows that the universal function $u_{1}=\left\{u_{1}(K) ; U_{1}\right\}$ exists.

\section{REFERENCES}

1. D. A. Buchsbaum, Some remarks on factorization in power series rings, J. of Math. and Mechanics, 10 (1961), 749-753.

2. E. D. Cashwell, C. J. Everett, The ring of number-theoretic functions, Pacific J. Math., 9 (1959), 975-985.

3. C. J. Everett, G. Whaples, Representations of sequences of sets, Amer. J. Math., 71 
(1949), 287-293.

4. P. R. Halmos, H. E. Vaughan, The marriage problem, Amer. J. Math., 72 (1950), 214-215.

5. N. Jacobson, Lectures in abstract algebra, D. Van Nostrand Co., N. Y. (1951) Ch. IV.

6. S. Lefschetz, Algebraic topology, Amer. Math. Soc. Colloq. Publ. XXVII (1942), p. 19.

7. H. Pollard, The theory of algebraic numbers, Carus Math. Monograph No. 9, J. Wiley and Sons, N. Y. (1950), p. 99.

8. P. Samuel, On unique factorization domains, Ill. J. of Math. 5 (1961), 1-17.

9. S. M. Ulam, $A$ collection of mathematical problems, Interscience Publishers, Inc., N. Y. (1960), p. 36 .

UNIVERSITY OF CALIFORNIA

Los Alamos, NeW Mexico 


\section{PACIFIC JOURNAL OF MATHEMATICS}

\section{EDITORS}

\section{RalPh S. Phillips}

Stanford University

Stanford, California

M. G. Arsove

University of Washington

Seattle 5, Washington
J. Dugundji

University of Southern California Los Angeles 7, California

Lowell J. Paige

University of California

Los Angeles 24, California

\section{ASSOCIATE EDITORS}
E. F. BECKENBACH
D. DERRY
H. L. ROYDEN
E. G. STRAUS
T. M. CHERRY
M. OHTSUKA
E. SPANIER
F. WOLF

\section{SUPPORTING INSTITUTIONS}

\author{
UNIVERSITY OF BRITISH COLUMBIA \\ CALIFORNIA INSTITUTE OF TECHNOLOGY \\ UNIVERSITY OF CALIFORNIA \\ MONTANA STATE UNIVERSITY \\ UNIVERSITY OF NEVADA \\ NEW MEXICO STATE UNIVERSITY \\ OREGON STATE UNIVERSITY \\ UNIVERSITY OF OREGON \\ OSAKA UNIVERSITY \\ UNIVERSITY OF SOUTHERN CALIFORNIA
}

STANFORD UNIVERSITY

UNIVERSITY OF TOKYO

UNIVERSITY OF UTAH

WASHINGTON STATE UNIVERSITY

UNIVERSITY OF WASHINGTON

AMERICAN MATHEMATICAL SOCIETY

CALIFORNIA RESEARCH CORPORATION SPACE TECHNOLOGY LABORATORIES

NAVAL ORDNANCE TEST STATION

Printed in Japan by International Academic Printing Co., Ltd., Tokyo Japan 


\section{Pacific Journal of Mathematics}

\section{Vol. 13, No. 1 \\ March, 1963}

Frantz Woodrow Ashley, Jr., A cone of super-(L) functions............. 1

Earl Robert Berkson, Some metrics on the subspaces of a Banach space....

Felix Earl Browder and Walter Strauss, Scattering for non-linear wave

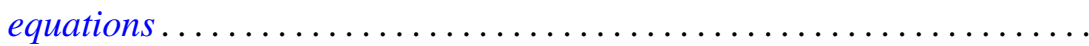

Edmond Darrell Cashwell and C. J. Everett, Formal power series ..........

Frank Sydney Cater, Continuous linear functionals on certain topological

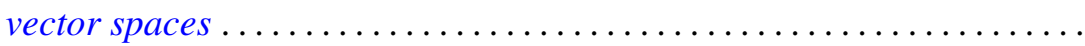

John Douglas Dixon, General group extensions ....................

Robert Pertsch Gilbert, On harmonic functions of four variables with

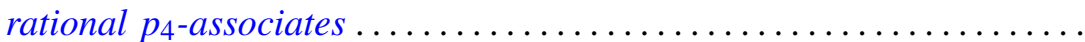

Irving Leonard Glicksberg, On convex hulls of translates ..............

Simon Hellerstein, On a class of meromorphic functions with deficient zeros

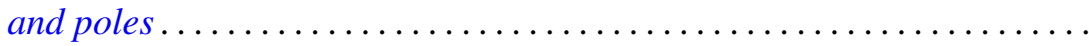

Donald William Kahn, Secondary cohomology operations which extend the

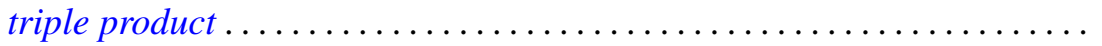

G. K. Leaf, A spectral theory for a class of linear operators .............

R. Sherman Lehman, Algebraic properties of the composition of solutions of partial differential equations ........................... 157

Joseph Lehner, On the generation of discontinuous groups ............. 169

S. P. Lloyd, On certain projections in spaces of continuous functions ...... 171 Fumi-Yuki Maeda, Generalized spectral operators on locally convex spaces ..................................

Donald Vern Meyer, $E^{3}$ modulo a 3-cell

William H. Mills, An application of linear programming to permutation groups.

Richard Scott Pierce, Centers of purity in abelian groups

Christian Pommerenke, On meromorphic starlike functions ...

Zalman Rubinstein, Analytic methods in the study of zeros of

polynomials...

B. N. Sahney, On the Nörlund summability of Fourier series

Tôru Saitô, Regular elements in an ordered semigroup . .

Lee Meyers Sonneborn, Level sets on spheres...........

Charles Andrew Swanson, Asymptotic estimates for limit point

problems .

Lucien Waelbroeck, On the analytic spectrum of Arens . .

Alvin (Murray) White, Singularities of a harmonic function of three

variables given by its series development .............

Kōichi Yamamoto, Decomposition fields of difference sets ...

Chung-Tao Yang, On the action of $\mathrm{SO}(3)$ on a cohomology manifold... 